\title{
Uretrocistografía en Niños: Percepción de los Padres
}

\author{
KARLA MOËNNE B ${ }^{1}$, CARLOS SAIEH A², XIMENA ORTEGA F ${ }^{1}$, \\ JUAN ANTONIO ESCAFFI $\mathrm{J}^{1}{ }$, CAROLINA PÉREZ $\mathrm{S} .{ }^{1}$ \\ 1. Radiólogo Infantil, Diagnóstico por Imágenes Clínica Las Condes. \\ 2. Nefrólogo Infantil, Departamento de Pediatría Clínica Las Condes.
}

\begin{abstract}
Uretrocystography in Children: Parents' Perception

Voiding cystourethrography (VCUG) has been usually consider as a painful and badly tolerated procedure in children. This opinion is not agree with local experience of the authors. Objective: To evaluate the parent's perception about VCUG in children before and after the procedure. Methods: In 2009, parents of children who came to Radiology Department for a VCUG completed an anonymous survey, including age and sex of their children, physician referent specialty, information received about the exam, expectation before and opinion after the procedure. Results: During 12 months 86 surveys were evaluated; patient's age was ranged between 1 month to 8 years with $52.3 \%$ (45) girls and $47.7 \%$ (41) males. Patients were referred from general pediatric in $59 \%$ or nephrology practice in $33 \% ; 20 \%$ of the patients did not receive any information about the procedure before. About the expectation of VCUG, previous the exam, parents considered the examination as a pediatrician visit in $2.3 \%$, uncomfortable in $26.7 \%$, a little painful in $16.3 \%$, painful in $21 \%$ and aggressive or terrible in $33.7 \%$. After the examination, parents' opinion was: $24.4 \%$ as a pediatrician visit, $45.3 \%$ uncomfortable, $22.1 \%$ a little painful, $4.7 \%$ painful and $3.5 \%$ aggressive or terrible. The global parents' perception about VCUG after the exam improved in $66 \%$ cases, did not change in $29 \%$ and went worse in $5 \%$. Conclusion: Parent's perception about VCUG in children significantly improves after the procedure; in $91.8 \%$ the examination was considered uncomfortable or only a little painful.

(Key words: Cystography, urinary catherization, children).

Rev Chil Pediatr 2010; 81 (4): 333-338
\end{abstract}

\section{RESUMEN}

Con frecuencia se califica a la uretrocistografía miccional (UCG) como un examen doloroso y mal tolerado por los niños, lo que resulta discordante con la experiencia de los autores. Objetivo: Evaluar la percepción de los padres de las UCG efectuadas a sus hijos, antes y después del examen. Durante el año 2009, en el Servicio de Radiología de Clínica Las Condes, se solicitó a los padres contestar anónimamente una encuesta que incluía: edad y sexo del niño, especialidad médico solicitante, información recibida respecto del examen, explicitar

Trabajo recibido el 29 de marzo de 2010, devuelto para corregir el 02 de junio de 2010, segunda versión el 27 de julio de 2010 , aceptado para publicación el 02 de agosto de 2010.

Correspondencia a:

Dra. Karla Moënne B.

E-mail:kmoenne@clc.cl 
expectativas antes del examen y opinión posterior. Se evaluaron 86 encuestas; edades: 1 mes a 8 años; 52,3\% (45) niñas y 47,7\% (41) varones; pediatra deriva 59\% y nefrólogo 33\%; 20\% no recibió información del solicitante. Antes del examen: $2,3 \%$ como una visita al pediatra, 26,7\% molesto, $16,3 \%$ un poco doloroso, $21 \%$ doloroso y $33,7 \%$ agresivo/terrible. Después de la UCG: $24,4 \%$ como una visita al pediatra, 45,3\% molesto, $22,1 \%$ un poco doloroso, $4,7 \%$ doloroso y $3,5 \%$ agresivo/terrible. Post-examen: $66 \%$ mejora la percepción, $29 \%$ mantiene y $5 \%$ empeora. Conclusiones: La percepción de los padres mejora significativamente después de efectuado el examen; el 91,8\% considera el examen como molesto o un poco doloroso.

(Palabras clave: Uretrocistografía miccional, cateterización vesical, niños).

Rev Chil Pediatr 2010; 81 (4): 333-338

\section{Introducción}

La uretrocistografía miccional (UCG) es un procedimiento habitual en el diagnóstico por imágenes en niños y uno de los más frecuentes entre los exámenes fluoroscópicos que se realizan en los departamentos de radiología pediátrica ${ }^{1}$. A pesar que la UCG se utiliza desde décadas, permanece como el estudio por imágen de elección para demostrar la anatomía y función del tracto urogenital inferior ${ }^{1,2}$. Su uso está indicado en el estudio de pacientes con variados cuadros clínicos que incluyen la infección del tracto urinario (ITU), pesquisa de relujo vésico-ureteral (RVU), dilataciones prenatales, anomalías renales congénitas, valvas de uretra posterior, hipospadias, malformaciones anorectales y trauma uretral, entre otras ${ }^{1,3}$.

La UCG se realiza instalando con técnica aséptica una fina sonda uretral (4-6F), a través de la cual se distiende la vejiga con solución de medio de contraste yodado hasta lograr micción espontánea, controlando el procedimiento con fluoroscopía pulsada ${ }^{1,4}$, que permite utilizar tiempos extremadamente breves de exposición a radiación ionizante. El examen es realizado habitualmente sin sedación ni anestesia general.

En la práctica clínica y en la literatura médica es posible observar opiniones variadas respecto a cómo toleran los niños este examen, la gran mayoría negativas, que van desde referir que se trata de un procedimiento que ocasiona escasas molestias a catalogarlo como doloroso e incluso "espantoso" 3,4 . Ha sido publicado que la UCG es el procedimiento de investigación urológica más estresante de todos los que se realizan en niños, en el que sobre el $27 \%$ de los pacientes experimentan severo distress, de acuerdo a la percepción de los padres y del staff que atiende a estos pacientes ${ }^{1}$. También existen reportes que recomiendan el uso de sedación para evitar el sufrimiento del niño y la ansiedad de los padres ${ }^{5-8}$. Sin embargo, la impresión existente en nuestro grupo de trabajo difiere de lo anterior y coincide con una visión menos negativa que publican otros autores, en el sentido de que después de explicar en detalle el procedimiento a los padres, acompañantes y al niño cuando corresponde, los padres evalúan el distress y dolor de sus hijos en la UCG como menor a lo que habían previsto, demostrando además que la ansiedad de los padres es un factor muy importante en esta percepción ${ }^{9}$.

El objetivo de nuestro trabajo fue conocer la percepción de los padres antes y después de realizar la UCG a sus hijos, además de evaluar la información recibida por los padres respecto del examen.

\section{Material y Métodos}

En el año 2009 (Enero a Agosto), en el Servicio de Diagnóstico por Imágenes de Clínica Las Condes, al finalizar las UCG efectuadas a niños, se solicitó a los padres contestar anónimamente una encuesta que incluía: edad y sexo del niño, especialidad del médico solicitante, entrega o no de información respecto del examen por el solicitante, radiólogo, secretarias o auxiliares de Rayos. Además, se pedía explicitar las expectativas del examen antes de efectuarlo y la opinión después de realizado, seleccionando una o más de las siguientes alternativas: como una visita al pediatra, mo- 
lesto, un poco doloroso, doloroso, agresivo/ terrible (figura 1). La encuesta consideraba un espacio final para comentarios o sugerencias.

Los exámenes se efectuaron con la técnica habitual: el niño y sus acompañantes fueron recibidos por una auxiliar de enfermería, quien solicitó el urocultivo comprobando su negatividad, otorgó información general al niño (cuando correspondía) y a quienes lo acompañaban, procediendo luego a efectuar el aseo genital requerido. Posteriormente, el radiólogo les explicó el procedimiento a realizar y procedió a instalar sonda fina en vejiga (habitualmente $\mathrm{N}^{\circ}$ 5), utilizando dimecaína líquida o vaselina estéril como lubricantes. Luego se procedió a llenar vejiga con solución de medio de contraste hidrosoluble, instalada en un porta-flebo a la mayor altura posible. $\mathrm{La}$ inyección se mantuvo en forma continua hasta lograr micción espontánea; en los niños menores de un año derivados por estudio de ITU, se efectuó de rutina un segundo llene cuando el

\section{ENCUESTA}

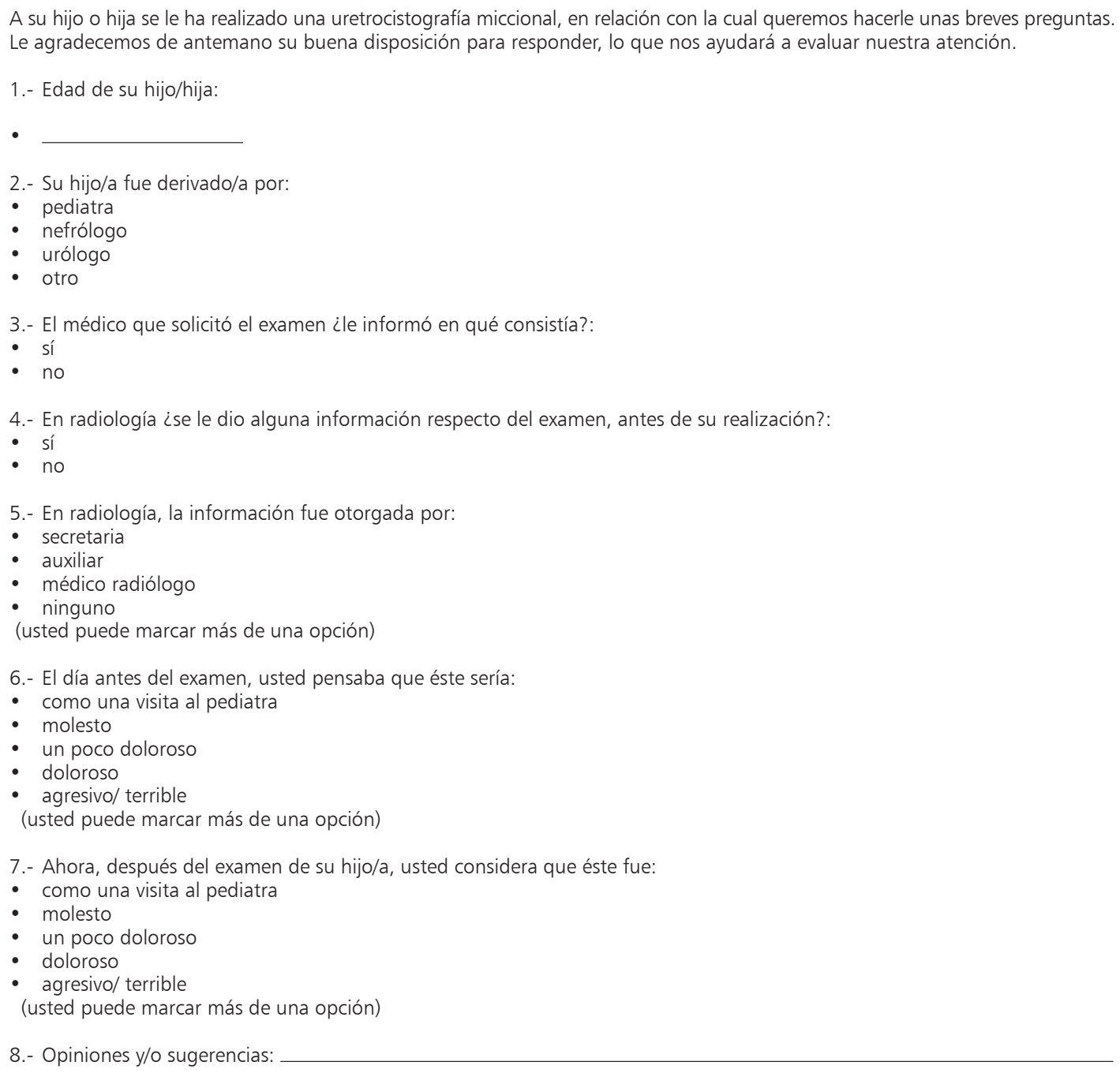

Figura 1. Encuesta para evaluación de la UCG según percepción de los padres. 
primer ciclo resultó normal. Durante el procedimiento se procuró distraer al niño con figuras adheridas al equipo, juguetes o administrando mamadera, cuando esto fue posible. Finalizada la micción, se procedió a retirar la sonda y a levantar el paciente desde la mesa, finalizando el examen. Con posterioridad, la auxiliar entregó la encuesta a los padres o acompañantes (figura 1), solicitándoles completarla en forma anónima.

En el análisis de las encuestas, cuando los padres marcaron más de una alternativa, se decidió considerar la peor de las opiniones, tanto antes como después del examen.

Durante el tiempo en que se desarrolló el estudio, los nefrólogos, urólogos y pediatras que indicaron las uretrocistografías no tuvieron conocimiento de éste, con el objeto de no modificar la conducta respecto de la información entregada a los padres.

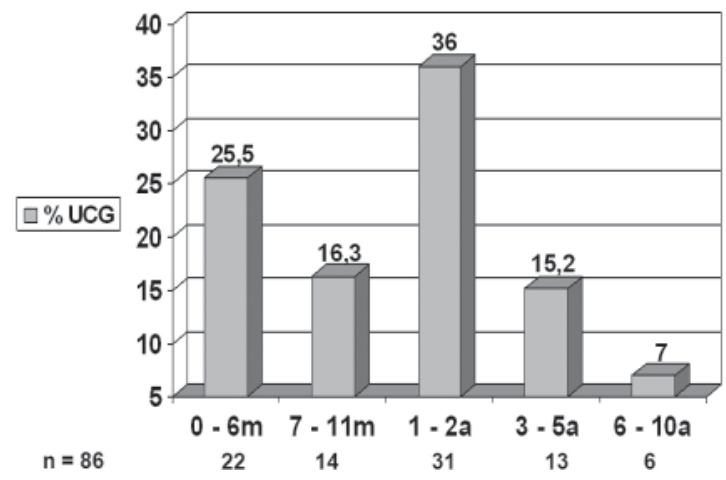

Figura 2. Gráfico de distribución de los pacientes estudiados según edad.

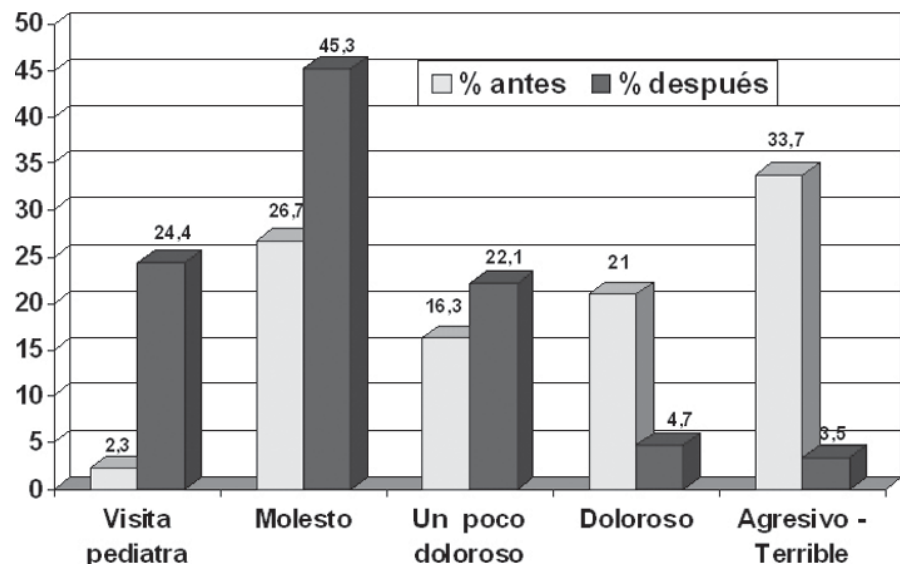

\section{Resultados}

En el período descrito se evaluaron 86 encuestas, que corresponden a la totalidad los pacientes a los que se solicitó llenar las encuestas, quienes lo hicieron sin inconvenientes.

Las edades de niños fluctuaron entre 1 mes y 8 años, distribuyéndose como se observa en la figura 2; el $41,8 \%$ corresponde a menores de 1 año. El 52,3\% (45) correspondió a niñas y $47,7 \%(41)$ a varones.

E1 $59 \%$ de los niños fue derivado por pediatras, 33\% por nefrólogos, $7 \%$ por urólogos y $1 \%$ por otro profesional. El $20 \%$ de los casos no recibió información respecto del examen de parte del solicitante y sólo en un paciente se refiere no haber recibido información en el Servicio de Radiología.

Antes del examen, la expectativa fue: $2,3 \%$ como una visita al pediatra, $26,7 \%$ molesto, $16,3 \%$ un poco doloroso, $21 \%$ doloroso y $33,7 \%$ agresivo/terrible. Después de efectuada la UCG, fue: $24,4 \%$ como una visita al pediatra, $45,3 \%$ molesto, $22,1 \%$ un poco doloroso, $4,7 \%$ doloroso y $3,5 \%$ agresivo/terrible. Después del examen: $66 \%$ mejora la percepción, $29 \%$ la mantiene y $5 \%$ empeora respecto de lo esperado (figura 3). El 49\% agregó comentarios positivos, $45 \%$ no escribió comentario alguno y $6 \%$ comentarios negativos, que correspondieron a deficiente atención en recepción, fundamentalmente retrasos.
Figura 3. Gráfico de barras muestra la comparación de las respuestas de los padres, incluyendo las expectativas de los padres antes del examen y las opiniones después de efectuado el examen. 


\section{Discusión}

El antecedente de ITU constituye la indicación más frecuente de estudio con UCG en la población pediátrica El manejo de esta patología, dirigido a prevenir o minimizar el daño renal y a evitar secuelas tardías, como la hipertensión arterial y la insuficiencia renal crónica, incluye el estudio por imágenes para la pesquisa de malformaciones y/o presencia de reflujo vésicoureteral. No existe consenso en relación a qué examen de imagen utilizar en cada paciente y por ésto existen múltiples protocolos en los distintos grupos de trabajo. En nuestro medio, el más utilizado consiste en estudiar con ultrasonografía renal/vesical y UCG a todo varón de cualquier edad o mujer menor de 5 años que presente una ITU ${ }^{10,11}$. En los últimos años, han aparecido publicaciones en relación con este tópico, que indican una tendencia a focalizar el estudio por imágenes en los niños que se identifiquen como de mayor riesgo, de modo de evitar distress y costo de exámenes, especialmente aquellos invasivos ${ }^{12,13}$.

La UCG miccional continúa siendo una gran herramienta diagnóstica en las imágenes pediátricas, tanto para evaluar la anatomía, especialmente la uretra en el varón, como para valorar configuración, capacidad y vaciamiento de la vejiga, además de pesquisar RVU. En variadas publicaciones se hace referencia a la UCG como un examen doloroso, mal tolerado y a veces terrible para los pacientes pediátri$\cos ^{1,3,4}$, llegando incluso a recomendar sedación, anestesia o hipnosis para evitar el sufrimiento del niño y la ansiedad de los padres ${ }^{5-8}$. Agrawalla et $\mathrm{al}^{1}$, refieren que la UCG es un examen difícil de realizar y una experiencia estresante para el paciente, su familia y el radiólogo.

Srivastava et $\mathrm{al}^{9}$, reconocen el temor de los niños y de sus padres frente a este examen y realizan un estudio prospectivo en un hospital de atención terciaria, en el que luego de explicar en detalle el procedimiento a los padres o acompañantes y al niño (cuando correspondía), demostraron mediante un cuestionario realizado posterior al examen que el temor y la ansiedad fueron mucho menores a lo esperado; también describen que la ansiedad de los padres se transmite al paciente y dificulta el estudio.

Es también nuestra experiencia que el conocer por adelantado los detalles del examen que se realizará al paciente facilita el procedimiento y logra mayor cooperación de niños y padres. Por esto, nuestra práctica habitual en el trabajo con niños incluye explicar en qué consiste cada uno de los procedimientos a los padres o acompañantes y a los niños, salvo cuando se trata de lactantes menores. En este trabajo, la evaluación de la percepción de los padres respecto de la UCG fue efectuada sin modificar la metodología habitual y los resultados obtenidos confirman que existe un importante cambio (positivo) en las expectativas respecto del examen y sólo un pequeño porcentaje $(8,2 \%)$ de los padres perciben que el procedimiento fue doloroso o agresivo-terrible para sus niños. También es importante mencionar que la mitad de los padres o acompañantes decidieron agregar comentarios positivos, que básicamente consistieron en agradecer la información entregada así como sentirse acogidos en el procedimiento y destacar el aspecto del escaso sufrimiento que el examen representó para el niño.

Existe bastante acuerdo en hacer todos los esfuerzos por disminuir el estrés que pueden significar los procedimientos invasivos a los niños, en este caso durante la ejecución de la uretrocistografías miccionales ${ }^{1}$. Nuestros resultados coinciden con la experiencia de otras series $^{9}$ en el sentido que el examen es mejor tolerado (en la práctica sin problemas por la mayoría de los niños) cuando disminuye el estrés de los padres y pacientes, lo que se logra muy especialmente aportando con anterioridad la adecuada información respecto del examen, creando así un ambiente de confianza y seguridad para el niño y sus padres. Algunos grupos citan a los padres con anterioridad ${ }^{1}$ para observar el procedimiento; es nuestra experiencia que en la mayoría de los casos es suficiente con las explicaciones entregadas antes de iniciar el examen si bien en forma ocasional existen padres en los que el nivel de estrés es mayor, por lo que se contactan con anterioridad para solucionar sus dudas y muchas veces solicitar sedación. Existen grupos que en la li- 
teratura recomiendan el uso de sedación o hipnosis para disminuir el estrés ${ }^{4,5}$; consideramos que los resultados obtenidos avalan nuestra percepción previa respecto de que la UCG es en general bien tolerada y resulta innecesario agregar cualquier tipo de sedación.

En resumen, los resultados obtenidos en la evaluación de la percepción de los padres respecto de la UCG confirman nuestra impresión previa, en el sentido que mayoritariamente la experiencia se evalúa en forma positiva.

Estimamos que el informar adecuadamente a los padres, acompañantes o pacientes (cuando corresponda), resulta de gran importancia para disminuir la ansiedad de éstos y lograr mayor colaboración. En este sentido, resulta fundamental que los pediatras conozcan cómo toleran los niños el procedimiento, ya que ellos cuentan con la confianza de los padres y de sus pacientes.

Agradecimientos: Los autores agradecen a las auxiliares de radiología, cuyo apoyo resulta fundamental para lograr los resultados obtenidos.

\section{Referencias}

1.- Agrawalla S, Pearce R, Goodman TR: How to perform the perfect voiding cystourethrogram Pediatr Radiol 2004; 34: 114-9.

2.- Palmer LS: Pediatric Urologic Imaging. Urol Clin N Am 33 (2006), 409-23.

3.- Ochoa C, Formigo F: Pruebas diagnósticas de imagen recomendadas en la infección urinaria. An Pediatr (Barc) 2007; 67 (5): 498-516.

4.- Butler L, Symons B, Henderson S, Shortliffe L, Spiegel $D$ : Hypnosis reduces distress and duration of an invasi- ve medical procedure for children. Pediatrics 2005; 115 (1): e77-e85.

5.- Stokland E, Andreasson S, Jacbsson B, Jodal U, Ljung $B$ : Sedation with Midazolam for voiding cystourethrography in children: a randomized double-blind study. Pediatr Radiol 2003; 33: 247-9.

6.- Akil L, Ozkol M, Ikizoglu OY, et al: Premedication during micturating cystourethrogram to achieve sedation and anxiolysis. Pediatr Nephrol 2005; 20: 1106-10.

7.- Green SM, Krauss B: Systemic. Analgesia and Sedation for Procedures. En: Roberts: Clinical Procedures in Emergency Medicine, 5th ed. [en línea] Elsevier. 2009. http://www.mdconsult.com/das/book/ body $/ 176177123-6 / 0 / 2083 / 280 \cdot$ html?tocnode $=577271$ 70\&fromURL $=280 . \mathrm{html} \# 4-\mathrm{u} 1.0$-B978-1-4160-36234..00033-X_1527 (consulta: 22-12,2009).

8.- Phillips D, Watson AR, Collier J: Distress and radiological investigations of the urinary tract in children. Eur J Pediatr 1996; 155: 684-7.

9.- Srivastava T, Betts G, Rosenberg AR, Kainer G: Perception of fear, distress and pain by parents of children undergoing a micturating cystourethrogram: a prospective study. J Paediatr Health 2001; 37: 271-3.

10.- De La Cruz J: Infección del tracto urinario en: Manual de Nefrología y urología pediátrica. Ed. Saieh C, Escala JM. Editorial Mediterráneo Santiago, Chile. $4^{\text {a }}$ ed. 2009; Cap 23 pag 127-31.

11.- Salas P, Álvarez E, Saieh C: Pautas de diagnóstico y tratamiento en infección urinaria. Rev Chil Pediatr 2003; 74 (3): 311-4.

12.- Marks S, Gordon I, Tullus K: Imaging in childhood urinary tract infections: time to reduce investigations. Pediatr Nephrol 2008; 23: 9-17.

13.- Muga O, Oñate E, Sota I, et al: Estudios por imagen en la primera infección urinaria con fiebre del lactante. ¿Es necesaria la cistografía miccional? An Pediatr (Barc) 2008; 69: 521-5. 